\title{
Active Primordial Bodies: Exploration of the primordial composition of ice-rich planetesimals and early-stage evolution in the outer solar system
}

Walter Harris (University of Arizona, Tucson)

e-mail: wharris@1pl.arizona.edu, phone: 520-621-6971

\section{Co-authors:}

Yanga R. Fernandez (University of Central Florida)

Gal Sarid (SETI Institute)

Jordan K. Steckloff (Planetary Science Institute, University of Texas at Austin)

Kat Volk (University of Arizona, Tucson)

Maria Womack (Florida Space Institute, University of Central Florida)

Laura M. Woodney (California State University, San Bernardino) 
Executive Summary: Active Primordial Bodies (APBS) are ice rich planetesimals orbiting or having perihelia in the giant planet region that experience outbursts or produce coma in the absence of water sublimation. These objects, including $\sim 20 \%$ of Centaurs and some long period comets, are minimally evolved compared with comets in the inner solar system: they represent a bridge population intermediate between pristine progenitors in the Kuiper Belt and Oort Cloud reservoirs and evolved inner solar system comets. The mechanisms responsible for APB behavior, the reasons why only some icy bodies are active, and how they evolve are not well understood. However, they are the least altered objects available for an exploration of the interior composition of a near-primordial body and a key window into planetesimal formation processes.

We advocate for a program of robotic exploration of APBs emphasizing a quartet of objects (29P/Schwassmann-Wachmann, 95P/Chiron, 174P/Echeclus, and P/2019 LD2) with characterized activity and outburst patterns, two of which are in active transition into the JFC population. The urgency of this opportunity is underscored by their near-term orbital characteristics which are favorable only for the next 20-30 years for missions in the New Frontiers and Discovery class. In addition, we recommend expanded surveys for activity across the giant planet region and deep study of a recently identified orbital transition region between the Centaur and Jupiter Family comet population.

1.0 Background: Ice-rich planetesimals are understood to be the remnant building blocks of the giant planets and the most pristine record of composition and conditions within the proto-solar disk. As such, they are our most direct path toward increasing our understanding of the processes governing how planetary systems form and evolve. The modern population of icy bodies (Fig. 1) consists of objects in dynamically stable reservoirs in the Oort Cloud (OC) and the Kuiper Belt (KB). From these reservoirs a small number of objects have been (and are continuously) perturbed inward toward the Sun, becoming long period OC and short period Jupiter Family comets (LPC, JFC) and the Centaurs orbiting between Jupiter and Neptune.

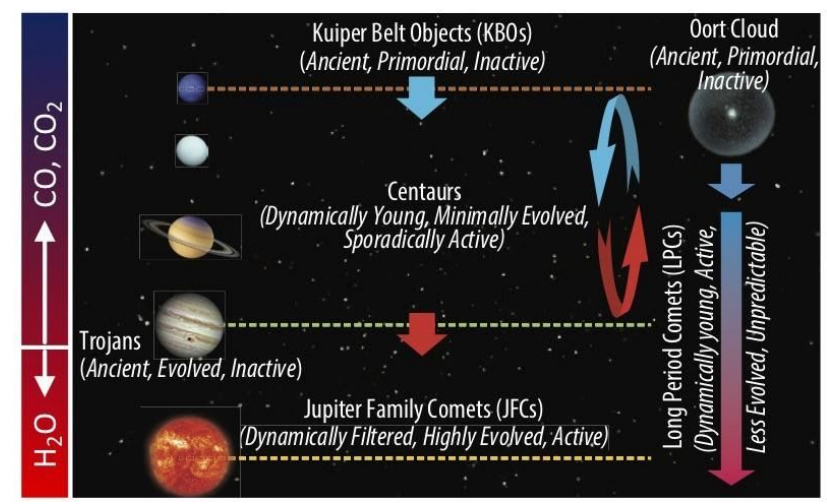

Figure 1. The different classifications of icy bodies including their dynamical and evolutionary states. Objects in the $O C$ and $K B$ reservoirs are the least evolved, but they are also inactive and remote. The $O C$ is the source region of the LPCs, while KB objects evolve through the Centaur region to become the JFCs. The extent of evolutionary processing increases as they experience more and closer encounters with the Sun.

The volatile composition, in particular the relative abundances, isotopic ratios, and chemical phases, of ice-rich planetesimals is our primary window through which to investigate the epoch of solar system formation. For bodies in the $\mathrm{OC}$ and $\mathrm{KB}$ this physical and chemical record is believed to be retained in its primordial form. Spectroscopy of surface ices can sample relative species abundances and place useful constraints on these bodies' internal structure and composition (e.g., Barucci et al. 2008; Brown 2012; de Bergh et al. 2013). However, nearly all of the minimally-altered bodies are too small, dark, and remote for detailed characterization with 
current Earth-based observatories, and those that can be observed have been affected by Gyr exposure to cosmic rays and stellar radiation (Stern \& Shull 1988; Strazzulla \& Johnson 1991). Instead, the vast majority of what we know of ice-rich planetesimal composition comes from observations of escaping gas and dust during active phases they experience as they approach the Sun. This activity is a consequence of physical evolution of the outer layers of a nucleus that accumulates as objects experience repetitive encounters with the Sun, gradually erasing their original surface and physical properties. Hence, observations of and encounters with objects that are newly active and have experienced less surface processing should most resemble their primordial state. Given their relative evolutionary state compared with comets, we refer to such objects as active primordial bodies (APBs).

Icy body activity can be broadly generalized into two categories: (1) sustained or 'quiescent' gas production that continues for extended periods and is typically greatest near orbital perihelion, and (2) stochastic 'outburst' events where large amounts of material are released in short intervals over a range of heliocentric distances.

Inner Solar System Activity: For comets well inside the orbit of Jupiter, quiescent activity is driven by sublimation that has a heliocentric dependence approximately following that of $\mathrm{H}_{2} \mathrm{O}$ ice that accelerates rapidly for objects inside of 3 au (e.g. Meech \& Svoren 2004). Comets in this region also produce significant quantities of $\mathrm{CO}_{2}$ and $\mathrm{CO}$, along with numerous minor species (e.g., $\mathrm{CN}, \mathrm{C}_{2}, \mathrm{NH}$ ). The ratio of the minor species to water have been shown to be relatively homogeneous across the region inside of 3 au for individual comets (A'Hearn \& Millis 1980) but cluster into production rate ratio classes with respect to each other (e.g., A'Hearn et al. 1995; Fink 2016). In contrast with quiescent activity, outburst activity for comets in the inner solar system is less well matched to a purely sublimation driven process. For many comets, the rate of outburst increases as they approach perihelion, but they can occur at any point along their orbit, with major events often occurring at distances where quiescent activity is low. Suggested mechanisms for outbursts include release of trapped gasses (Belton et al. 2008), landslide events (Steckloff et al. 2016), and amorphous-crystalline ice transformation (Prialnik \& Bar-Nun 1992).

Outer Solar System Activity from APBs: The mechanism(s) of quiescent activity in the outer solar system beyond 5 au are significantly less well understood, particularly for icy bodies that are not DN and do not enter the region of water sublimation. The first object shown to be active in an orbit entirely beyond Jupiter was 29P/Schwassmann-Wachmann 1 (SW1), which was discovered in 1927 while in outburst (e.g., Berman \& Whipple 1928) and has been continuously active over nearly a century of subsequent observations. Additional active bodies were discovered over time, as larger telescopes enabled the discovery and characterization of the Centaurs, the KB, and the more remote portion of LPC orbits. By the end of the $20^{\text {th }}$ century, $\sim 20$ active objects with perihelia beyond 5 au had been characterized including both Centaurs (e.g., SW1 and 95P/Chiron) and LPCs. In addition to these, several other comets with smaller perihelia (e.g., 1P/Halley and C/1995 O1 Hale-Bopp) had been observed to be active in the outer solar system. Due to their distance, detection of volatile production is challenging, and activity is typically detected by proxy from a dust coma. Gas production has only been directly measured in a few cases, including detection of CO emission from SW1 (Senay \& Jewitt 1994) and Chiron (Womack \& Stern 1999) and several species from Hale-Bopp (Biver et al. 1997).

With the possible exception of DN LPCs, which may retain a primordial surface layer of super-volatile ices, the source of outer solar system quiescent activity is inconsistent with 
sublimation. Water sublimation drops off rapidly beyond $\sim 5$ au (e.g. Biver et al. 2002) and cannot explain the observed activity beyond 7 au seen at Chiron, Hale-Bopp, and other Centaurs and LPCs. Other, more volatile species, such as $\mathrm{CO}, \mathrm{CO}_{2}$, and even $\mathrm{CH}_{4}$, have been proposed as a source, but their sublimation temperatures (Meech \& Svoren 2004) are a poor match to the observed heliocentric trends for active objects. While $\mathrm{CO}_{2}$ sublimation can contribute to activity out to $\sim 20 \mathrm{au}$, it has not been detected in the most active outer solar system body, SW1 (Ootsubo et al. 2012). $\mathrm{CO}$ and $\mathrm{CH}_{4}$ are super-volatile across the entire giant planet region. While both could contribute to DN LPC activity, neither are stable as ices on KB objects out to 50 au that are the progenitor population of the Centaurs. Another potential candidate is annealing and crystallization of amorphous ice (e.g. Prilanik et al. 2004), which should occur progressively over the giant planet region and is consistent with similar heliocentric production profiles of minor species observed from LPC Hale-Bopp (Biver et al. 2002).

In addition to quiescent activity, APBs in the outer solar system also experience outbursts over a wide range of heliocentric distances and are even further out of apparent solar energy equilibrium than their inner solar system counterparts. Significant examples include 1P/Halley, which underwent a $5 \mathrm{M}_{\mathrm{V}}$ outburst at a heliocentric distance $>14$ au (West et al. 1991; Prialnik \& Bar-Nun 1992), outbursts of Hale-Bopp between 6-7 au (Sekanina 1997), sustained outbursts from Chiron at distances $>10$ au (Hartman et al. 1990; Bus et al. 2001), and 174P/Echeclus with a $7 \mathrm{M}_{\mathrm{V}}$ outburst at 13 au (Bauer et al. 2008). However, none of these compare with the case of SW1, which outbursts more often than any other object and has experienced multiple events of 1-5 $\mathrm{M}_{\mathrm{V}}$ each year since its discovery (e.g., Trigo-Rodríguez et al. 2008; Miles et al. 2016).

Significance: Activity and physical evolution of APBs in the outer solar system has been evident, even prior to the previous Decadal Survey. With increasing observations and observed sample size, and greater diversity in types of objects, we gain more insights into this active evolutionary state. The nature of this activity is consistent with different mechanisms and sublimation sources compared to inner solar system comets, which are in the water-dominated activity region. This strongly suggests that with APBs we are seeing an earlier evolutionary state, as precursors for active comet nuclei. This framework promotes APBs as key exploration targets, particularly in comparison to the JFCs targeted by previous space missions. Furthermore, being in an intermediate evolutionary state, between the cold and mostly inert KB and the active and processed JFCs, the diverse grouping of APBs can be identified as a bridge between reservoir objects and comets. As such, the study and exploration of APBs provides deeper and expanded insight into the conditions of planetary formation and small body evolution.

2.0 A Decade of Discovery: The decade since the publication of Visions and Voyages has been marked by a series of transformative advances in our understanding of: (1) The extent, characteristics, and diversity of outer solar system activity; (2) The orbital and evolutionary pathways that icy bodies follow into the JFC population; (3) The importance of APBs exploration for bridging the physical differences between reservoir and cometary end-state.

Expanding Numbers and Taxonomy: Steady improvements in the depth, wavelength range, and coverage of survey capabilities have greatly extended our ability to detect and characterize APBs and their local environments over increasing heliocentric distance ranges. As of mid-2020, the total number of known APBs is approaching 200 objects, including $>30$ active Centaurs and LPCs with activity at $>20$ AU (Szabó et al. 2008; Jewitt et al. 2017). These results underscore the 
surprising ubiquity of APBs throughout the giant planet region, the diversity in activity profiles, and their complex local environments. Key discoveries over the previous decade include:

- $10-20 \%$ of all Centaurs are at least sporadically active, with the presence of detected activity correlated with smaller perihelia between 5-12 au (Jewitt 2009; Peixinho et al. 2020).

- While the number of direct detections of $\mathrm{CO}$ emission from Centaurs remains small (e.g. SW1, Hale-Bopp, 95P/Chiron, and 174P/Echeclus), the accumulated data has shown variability $(>10 \mathrm{x})$ in area-averaged production of objects at similar heliocentric distance and substantial deviation from the power law heliocentric distance activity relationship followed by Hale-Bopp (Womack et al. 2017).

- Stellar occultation measurements have identified complex grain environments near the larger active Centaurs, including a stable ring system around 10199 Chariklo (Braga-Ribas et al. 2014) and several rings or dust shrouds surrounding 95P/Chiron (Sickafoose et al. 2020).

- Activity monitoring of active LPCs has shown differences in heliocentric distance brightening profiles that track with the distance where activity is first detected and additional evidence that DN LPCs are brighter than returning objects (Sárneczky et al. 2016)

- Comparison of the Dust: $\mathrm{CO}+\mathrm{CO}_{2}$ ratios shows distinct differences between the activity patterns of outer solar system LPCs and LPCs and JFCs inside of 4.5 au (Bauer et al. 2015)

Spacecraft Encounters: Two encounter missions in the previous decade provided essential context for the importance of active Centaurs to our understanding of primordial volatile characteristics and the physical state of comets relative to their progenitor populations:

- In 2014, the Rosetta spacecraft entered orbit around 67P/Churyumov-Gerasimenko. Over the next 2 years it monitored activity patterns, measured volatile production, and mapped the surface as 67P approached perihelion. This documented the eroded state and resurfacing rate of 67P (El-Maarry et al. 2016). Observations of water and $\mathrm{CO}_{2}$ production identified activity heterogeneity similar to that seen from 103P/Hartley (Feaga et al. 2007). They also revealed variability caused by seasonal illumination patterns, with sublimation from different depths below the surface altering the ratio of different volatiles in the coma (Fink et al. 2016).

- The flyby encounter of the $2014 \mathrm{MU}_{69}$ (Arrokoth) in the KB by the New Horizons spacecraft (Stern et al. 2019) revealed both bulk similarities between it and 67P and drove home the extent to which long residency in the inner solar system profoundly alters the primordial characteristics of the JFCs (Fig. 2).

The profound differences between these two objects that bookend the evolution of small icy bodies underscores the significance of exploring physical and activity characteristics of Centaurs as evolutionary bridges between the KB and JFCs.

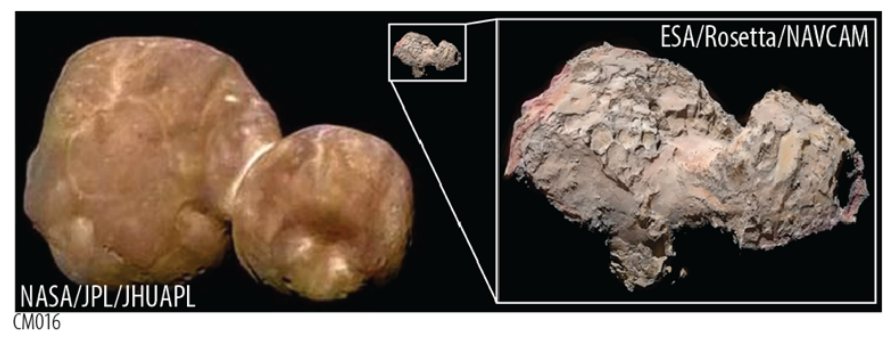

Figure 2. The relatively featureless properties of $2014 \quad M U_{69}$ (Left) are compared with the heavily eroded surface of $67 P$ (Right, shown to scale at center).

A Dynamical Gateway: Recent dynamical modeling revealed an underlying order within the chaotic transition from the outer to inner solar system. Sarid et al. (2019) identified a dynamical 
path facilitating the transition between Centaurs and JFCs: a short-lived $\left(\sim 10^{3} \mathrm{yr}\right)$, low-eccentricity 'gateway' orbit just beyond Jupiter (between roughly 5.4 and 7.8 au) through which over $70 \%$ of Centaurs that enter the JFC population pass just prior to entering the inner solar system (Fig. 3). The most prominent member of the gateway orbit is SW1, which is also the most active known APB and has a 40\% likelihood of transitioning to the JFC population in the next few thousand years. SW1 shares the gateway with several other transitioning Centaurs including two APBs, P/2010 TO20 LINEAR-Grauer and the recently discovered P/2019 LD2. The latter is an object of high significance because, in addition to its activity, it has a $>90 \%$ chance of becoming a JFC after a close encounter with Jupiter in the early 2060s (Kareta et al., 2020, Steckloff et al., 2020).

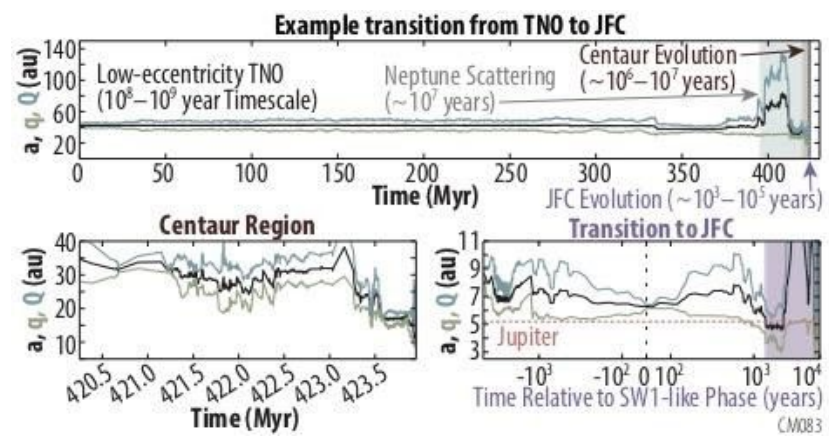

Figure 3. A sample simulation shows the path of an individual $K B$ object (top) into the Centaur region and from there (lower left) through the gateway and into the JFC population (lower right). The gateway-JFC transition timescale is $<0.01 \%$ of the median Centaur lifetime.

The short time between entering the gateway and a transition to the JFCs makes Centaur ABP behavior a common element of comet evolution. Gateway Centaurs are embryonic JFCs with characteristics that are directly relatable to the evolutionary processing from TNO to comet.

3.0 Scientific Objectives: APB's offer a unique opportunity to probe the composition and formation of small outer solar system bodies. Unlike exploration of inactive bodies (e.g TNOs), where only surface composition can be probed, exploration of APBs offers an opportunity to probe interior composition. The lack of a long history of activity (such as for JFC) also ensures that the composition being probed is as close as possible to primordial. This information will directly yield insights into the conditions in the planetesimal disk where they formed; a better understanding of early activity and how activity changes as objects evolve to smaller heliocentric distances will also allow us to put the activity of more evolved objects into better context. Table 1 summarizes the exploration objectives presented for APBs.

4.0 The Need for Immediate Action: A combination of factors argues for emphasizing the development of missions to explore APBs and, in particular, active Centaurs. Of the Centaurs known to be active, a small number, including 29P/SW1, 95P/Chiron, 174P/Echeclus, and $\mathrm{P} / 2019$ LD2, stand out by virtue of the magnitude of their activity, their outburst patterns, and/or their near-term orbital evolution. Fortuitously, each of these objects are currently occupying or approaching favorable orbital configurations for exploration over the next 20 to 30 years. SW1, the most active Centaur, currently occupies a low eccentricity orbit just beyond Jupiter, but its 2038 opposition with Jupiter will increase its eccentricity and may alter its well-characterized pattern of repetitive outbursts. The sporadically active and outbursting Centaurs 95P/Chiron and $174 \mathrm{P} /$ Echeclus will next pass within the orbit of Saturn in the early 2040s on their way to perihelia later that decade. Centaur-in-transition P/2019 LD2 will experience close encounters with Jupiter in 2027 and 2063 after which it has a $>90 \%$ chance of becoming a JFC (Kareta et 
al., 2020). Statistically, it is quite rare for an object as large as P/2019 LD2 (H mag 12.2) to undergo this transition, and it therefore provides an unprecedented opportunity to study in-situ a small body in both its APB and comet activity phases.

Outer solar system encounters and rendezvous missions to active APBs inside the orbit of Saturn typically require $>10$ year cruise times with conventional spacecraft. It is therefore essential that missions to key objects be launched within the next 10-15 years in order to meet the deadlines imposed by their orbital characteristics. This urgency argues for the immediate development of concepts to explore them and the inclusion of APBs in the New Frontiers mission line over the next decade. Should this series of opportunities be lost the earliest returning objects will be available is the late 2080s. In the case of P/2019 LD2, the opportunity to monitor a JFC transition event will be lost entirely.

Table 1. Exploration Objectives for APBS

\begin{tabular}{|c|c|c|}
\hline Question: & Goal: & Exploration Objective: \\
\hline \multirow{7}{*}{$\begin{array}{l}\text { What physical } \\
\text { mechanisms drive } \\
\text { activity and } \\
\text { evolution of ice-rich } \\
\text { planetesimals } \\
\text { beyond } 5 \text { au? }\end{array}$} & \multirow{2}{*}{$\begin{array}{l}\text { Understand how presolar } \\
\text { processes are recorded in } \\
\text { the materials of primitive } \\
\text { bodies }\end{array}$} & $\begin{array}{l}\text { Measure the relative composition and isotopic signature of escaping } \\
\text { volatiles }\end{array}$ \\
\hline & & $\begin{array}{l}\text { Map the distribution, local environment and energy balance of active } \\
\text { regions on APB surfaces }\end{array}$ \\
\hline & \multirow{2}{*}{$\begin{array}{l}\text { Probe accretion and } \\
\text { formative processes in } \\
\text { the solar nebula }\end{array}$} & $\begin{array}{l}\text { Identify the features and morphology of real-time physical } \\
\text { modification of APB nuclei }\end{array}$ \\
\hline & & $\begin{array}{l}\text { Investigate early stage evolutionary processes associated with Centaur } \\
\text { transition through the gateway region }\end{array}$ \\
\hline & \multirow{3}{*}{$\begin{array}{l}\text { Determine the effect and } \\
\text { timing of secondary } \\
\text { processes on the } \\
\text { evolution of primitive } \\
\text { bodies }\end{array}$} & $\begin{array}{l}\text { Explore the mechanisms, compositional distinctiveness, and energetics } \\
\text { of outbursts }\end{array}$ \\
\hline & & $\begin{array}{l}\text { Probe the interior structures of the near-subsurface and deep interior of } \\
\text { APBs }\end{array}$ \\
\hline & & $\begin{array}{l}\text { Compare the activity patterns and evolution of APBs in different } \\
\text { dynamical classes }\end{array}$ \\
\hline
\end{tabular}

5.0 Recommendations: We recommend that R\&D be put towards multi-faceted, complementary, explorations of ice-rich and active primordial bodies to uncover the rich network of ways that APBs can be used to explore formation and evolution in the solar system.

Survey and Characterization: Key (synoptic and multiwavelength) observations of Centaurs and LPCs at a range of heliocentric distances will allow a better understanding of these icy bodies' evolutionary processes. These observations have heretofore been difficult because of the general faintness of many of these objects, but such studies are vital in order to have an unbiased picture of Centaur and LPC properties and behaviors. For Centaurs this means studies of objects that have recently left the Kuiper Belt, others that are embedded in the giant-planet region, and still others that soon will become JFCs. While active Centaurs should be prioritized, ostensibly-inactive ones should also be monitored for transient eruptions and for (as, e.g., in the case of P/2019 LD2) the initiation of long-term active episodes. For LPCs, this means studies of 
both new and old objects, and includes Damocloids that might at first glance appear to be inactive. Long-term dedicated and coordinated observations from amateur and professional astronomers are requested to characterize the nuclei and dust environments via photometry, lightcurves, broadband filter imaging, astrometry, spectroscopy, and occultations. Furthermore, the community should take advantage of observing opportunities provided by major telescopic facilities such as LSST, JWST, NGRST, and VRO. Such observations will allow unprecedented assessments of Centaur and LPC nuclei, dust, and gas. For example, we will learn about the presence of moonlets or ring systems; about overall mass loss; and about the relative abundances of major volatiles (e.g. $\mathrm{CO}, \mathrm{CO}_{2}, \mathrm{H}_{2} \mathrm{O}$ ) and organics, and their mixture within nuclei.

Laboratory: We advocate for the following activities, as supporting exploration and advancing basic scientific knowledge: Develop techniques to measure combined thermo-mechanical properties of solid ice phases for water ice and other volatile molecular species $\left(\mathrm{CO}, \mathrm{CO}_{2}\right.$, $\mathrm{CH}_{3} \mathrm{OH}, \mathrm{HCN}, \mathrm{CH}_{4}$, etc.); Measure thermodynamic properties of porous and icy materials and response to static and dynamic pressures; Develop measurement techniques for amorphous and clathrate hydrate states of water ice, specifically with regards to retaining and releasing hyper-volatile molecular species $\left(\mathrm{CO}, \mathrm{N}_{2}, \mathrm{O}_{2}, \mathrm{CH}_{4}\right.$, etc.), and the dependency on deposition conditions (gas compositional ratios, temperature and pressure); Measure the sublimation behavior of volatile molecular species, under cryogenic conditions; Measure and develop spectral libraries to encompass ice-rich mixtures under pristine and evolved conditions.

Missions: We advocate for a robust series of missions targeting APBs, with emphasis on targets of special significance described in Section 4 for which rapid development is required. Previous concept studies for exploring the active Centaurs (e.g. Chiron) were developed at the New Frontiers class, and we recommend adding $A P B S$ as an opportunity in the upcoming call. Achieving the full set of objectives described in Table 1 will require in situ exploration. We advocate for mission types including: (1) Active Centaur flyby or tour; (2) Active Centaur orbital explorer; (3) Remote LPC interceptor; (4) Active Centaur coma or surface sample return.

At the Discovery class, opportunities include individual flybys and special configurations that allow for orbital exploration or tours of active Centaurs. At the New Frontiers class, in situ exploration of remote active Centaurs will require electric propulsion and nuclear power to enable safe operation at large heliocentric distance in a complex grain environment. LPC interceptor would require rapid deployment following discovery of a highly active object at large heliocentric distance that can be encountered between 5-8 au. This should be developed with a secondary target as a backup. Active Centaur cryogenic sample return may be possible within New Frontiers with a minimal payload, but is more likely at the Flagship class. Relevant APBs (P/2019 LD2, SW1, 2010 LINEAR-Grauer) may be accessible and will be more pristine and volatile rich than any JFC target.

An orbital rendezvous with an active Centaur would have the possibility of dramatically transforming our understanding of icy planetesimals much like Rosetta transformed our understanding of comets. New Frontiers to Flagship class missions would open the door to the possibility of visiting and characterizing multiple high-priority targets, perhaps with a combination of flybys and orbital rendezvous.

References: $\underline{\text { https://ui.adsabs.harvard.edu/public-libraries/ESHsjD7eQa6n1xZq1KC }}$ 